\title{
Impacts of Stock Indices, Oil and Twitter Sentiment on Major Cryptocurrencies during the COVID-19 First Wave
}

\author{
Nikolaos A. Kyriazis ${ }^{1}$
}

\begin{abstract}
This paper sets under scrutiny whether the S\&P500, oil, and Twitter-based uncertainty about financial markets affect the returns and volatility of three major cryptocurrencies. Estimations are conducted concerning Bitcoin, Bitcoin Cash, and Dogecoin during the first wave of the COVID-19 pandemic. Findings document that Twitter uncertainty exhibits a weaker impact on cryptocurrencies than the S\&P500 and crude oil. S\&P500 constitutes a positive and significant determinant while impacts of oil are weaker and mixed. The volatility of cryptocurrencies is found to display a non-linear character. Moreover, it is revealed that Dogecoin could be more useful to investors as a speculative tool than Bitcoin and Bitcoin Cash. These outcomes inform the interested reader that traditional investments are influential in a much larger degree towards modern financial assets than investor sentiment when economic conditions are stressed.
\end{abstract}

JEL classification numbers: E7, F3, G1

Keywords: Twitter Sentiment, Stock, Oil, Cryptocurrency, COVID-19 pandemic.

\section{Introduction}

Modern forms of liquidity and investments have been at the epicenter of heated academic debates since their outstanding bull market in 2017. This has generated a proliferating bulk of relevant academic work (Yermack, 2015; Ammous, 2018; Beneki et al., 2019; Corbet et al., 2019; Fassas, A. et al., 2020; Papadamou et al., 2021) in order to cast light on unknown aspects of these risky and sophisticated assets and help the academic community, investors and policymakers to acquire a multi-spectral view on this hot topic. Notably, the boost in relevant financial press articles rests largely upon the fact that an increasing number of risky investors are attracted by the high profitability engendered by large fluctuations in cryptocurrency returns.

Digital forms of money and investment prove to be far from predictable and the large extent of inefficiency in their market is found to lead to bubble formation and growth. The latter remains fed by irrational behaviour and mimicking phenomena among misinformed and/or speculative investors. Such a persistent tendency towards investing in cryptocurrencies is significantly reinforced by stressed economic conditions in a worldwide level due to bear markets brought about in a global scale. Bad performance of traditional financial assets has urged monetary authorities to employ very lax monetary policies and provide ample liquidity infusions and has resulted even in negative interest rates. The lack of noteworthy levels of returns for investors has been crucial for rendering them more favourable towards cryptocurrency markets (Kyriazis et al., 2020).

${ }^{1}$ Department of Economics, University of Thessaly, Greece

Article Info: Received: June 2, 2021. Revised: August 17, 2021. Published online: September 7, 2021. 
Bitcoin was invented in 2008 by Satoshi Nakamoto and is a decentralized digital currency, with no central authority. Bitcoin functions by a peer-to-peer bitcoin network and there is no need for intermediaries so is expected to reduce transaction costs if it succeeds in becoming adopted in a worldwide level. Cryptography is employed to verify transactions via network nodes and all transactions are recorded in a public distributed ledger, the "blockchain". The supply of Bitcoins is capped to 21 million units according to its protocol. This has generated high tendencies for bubble creation regarding the market values of Bitcoin and this is the reason why it is considered to be a speculative asset (Böhme et al., 2015; Cheah and Fry, 2015; Blau, 2018). Bitcoin and cryptocurrencies in general are also considered to be means for illegal transactions (Foley et al., 2019) and this renders more difficult the way towards their legalization and acquiring the "legal tender" by monetary authorities.

Bitcoin Cash is tightly connected with Bitcoin as it constitutes a fork of Bitcoin that was created in 2017. In November 2018, two cryptocurrencies came to the surface when Bitcoin Cash was split, which were: a) Bitcoin Cash and b) Bitcoin SV. It should be noted that Bitcoin Cash has the benefit over Bitcoin that it enables more transactions per second to be processed ${ }^{2}$. When Bitcoin Cash traded for the first time, on 1 August 2017, its market value was approximatelly 240 US dollars due to the high levels of market values (almost 2,700 US dollars) that Bitcoin had already succeeded.

Dogecoin was created by the software engineers Billy Markus and Jackson Palmer in order to serve as a payment system that provides high speed, is easy to use and needs no financial intermediation. It started trading in late 2013 and since the beginning it exhibited bubble tendencies due to high demand from investors. Dogecoin is steadily among the highest-capitalized cryptocurrencies. Its capitalization nowadays exceeds 5 billion US dollars ${ }^{3}$. It is considered to be very volatile and has gained popularity among investors as its low price makes it accessible to a large mumber of economic agents. Even though it was initially planned to have its supply capped, this restriction was later removed in order to be able to keep pace with inflationary conditions. Dogecoin has lately (February 2021) been among the major cryptocurrencies that have received support by Elon Musk (similarly with Bitcoin) via Twitter and this has rendered it even more popular than before to investors that seek high profit ${ }^{4}$.

Investigating the determinants of returns and volatility in cryptocurrency markets has been at the focus of a large and constantly increasing bulk of academic papers. The present study examines whether the highly innovative sentiment index that is based on tweets is influential on cryptocurrency performance. To be more precise, the Twitter-based Market Uncertainty (TMU) Index by Renault et al. $(2019)^{5}$ has been employed. Moreover, the impacts of traditional assets such as the well-established S\&P500 representative stock index and crude oil are under scrutiny in this study.

Econometric estimations have taken place in order to identify the form of linkages between Twitter-based uncertainty, the S\&P500, crude oil and the returns and volatility of three major cryptocurrencies that enjoy high capitalization and high trading volume. More specifically, the Bitcoin, Bitcoin Cash, and Dogecoin cryptocurrencies are investigated that are considered to be among the drivers of bull or bear tendencies in the overall market of digital currencies. In this study the task of investigating cryptocurrency performance during the first wave of the COVID-19 pandemic is undertaken. Thereby, the period under scrutiny spans from 22 January 2020 until 15 September 2020. By adopting advanced GARCH specifications, we shed light on the character of cryptocurrency returns' responses to alterations in investor sentiment (as measured by tweets) and the returns of highly important traditional assets (the S\&P500 and crude oil). The S\&P500 index is considered to be among the stock indices that are

\footnotetext{
${ }^{2}$ https://www.theverge.com/2018/4/12/17229796/bitcoin-cash-conflict-transactions-fight

${ }^{3}$ https://coinmarketcap.com/currencies/dogecoin/

${ }^{4} \mathrm{https} / / / \mathrm{www}$.bloomberg.com/news/articles/2021-02-07/dogecoin-soars-again-toward-record-after-crashing-last-week

${ }^{5} \mathrm{https}: / / \mathrm{www}$.policyuncertainty.com/twitter_uncert.html
} 
representative of stock markets in a worldwide context and is widely adopted in empirical research (Papadamou et al., 2019; Balcilar et al., 2020; Baig et al., 2021).

To the best of our knowledge, this is the first study to examine the connection of these determinants on these specific leading cryptocurrencies with these exact methodologies. More specifically, we cast light on the interlinkages among traditional and modern financial assets and measures of uncertainty that are considered to be a breakthrough in economics and finance tools. This takes place through the prism of the harsh consequences of a health crisis that has quickly been transformed into a financial crisis in a worldwide level.

The remainder of this study is structured as follows. Section 2 provides academic literature related with the nexus between Twitter-based sentiment and cryptocurrencies, the linkages of the COVID19 pandemic with cryptocurrencies, the connection between COVID-19 and stock markets as well as how this disease influences commodities and precious metals. Section 3 presents the data downloaded and the methodologies adopted for the purposes of estimations. Furthermore, Section 4 displays and analyzes the empirical outcomes and provides a discussion of their economic implications. Finally, Section 5 concludes and suggests avenues for further research.

\section{Literature review}

An impetus but proliferating level of interest by academics, investors and the financial press has aroused regarding the connection of Twitter sentiment and financial assets with the COVID-19 disease. This comes to augment the also nascent but highly increasing number of empirical studies investigating the nexus of COVID-19 with modern financial assets -such as cryptocurrencies- and traditional financial assets such as bonds, stocks, currencies and commodities. The present study covers four strands of the literature related with the COVID-19 pandemic and cryptocurrencies.

Studies with meaningful findings about the connection between Twitter-based sentiment and cryptocurrencies include Karalevicius et al. (2018) Baig et al. (2019), Eom et al. (2019), Philippas et al. (2019) and Shen et al. (2019). Furthermore, among latest relevant research the studies of Ibikunle et al. (2020) and Guegan and Renault (2020) are brought to the surface. More specifically, Karalevicius et al. (2018) use Bitcoin-related news articles and blog parts and assign positive and negative values of sentiment in order to investigate the linkage between media sentiment and Bitcoin prices. Sentiment analysis deriving from natural language processing techniques and based on lexicon approaches indicates that prices follow the sentiment. Nevertheless, due to the market overreacting a little the price moves to make a correction. Late movers finally push the market value towards the sentiment direction again. Overall, it is argued that signals cannot help the interested trader to outperform the market. Moreover, Baig et al. (2019) employ a number of alternative measures of Bitcoin-level and market-wide sentiment in order to study the unusual level of Bitcoin price clustering. By adopting the Huang et al. (2015) and the Baker and Wurgler sentiment indices and intra-day transaction-level data, the round clustering, strategic clustering and total clustering measures are used. Correlations and Ordinary Least Squares (OLS) estimations reveal the existence of a robust positive linkage between price clustering and the sentiment indices employed. To be more precise, higher sentiment as measures by Google Trends one standard deviation explains about $1 / 40$ to $1 / 20$ of the unusual price clustering in Bitcoin.

From their perspective, Eom et al. (2019) look into the statistical features and how predictable are Bitcoin returns and volatility. The Google Trends Index (GTI) is adopted to represent investor sentiment. Autoregression models are employed for examining predictability. Findings indicate that investor sentiment influences the level by which returns and volatility can be predicted in future periods and that Bitcoin is influenced by investor behaviour. This gives credence to the argument that Bitcoin is a risky investment asset rather than a currency. When it comes to Shen et al. (2019), they examine the impacts of 
investor attention on Bitcoin returns, trading volume and realized volatility. Tweets in Twiitter are employed as a measure of attention. Linear causality (Granger, 1969) but also non-linear Granger causality (Diks and Panchenko, 2006) procedures are adopted in Vector Autoregressive (VAR) models. It is argued that tweets during previous days are determinants of Bitcoin realized volatility (RV) and volume but do not significantly influence returns. Thereby, tweets can predict RV and volume in a reliable manner. By splitting into sub samples, they provide evidence that in more recent years tweets have become more influential towards realized volatility. In a partly simiar view, Philippas et al. (2019) investigate whether signals from Twitter and Google Trends are determinants of the jumps in Bitcoin price behaviour. A bivariate VAR-X framework is adopted for estimations while a Levy process is used to model Bitcoin values. The jump diffusion model of Kou (2002) is employed. Empirical outcomes present that media networks exert only partial impacts on Bitcoin market values. This phenomenon is more intense during turbulent periods when uncertainty is higher.

In a somewhat different vein, Ibikunle et al. (2020) decompose Bitcoin market values into efficient and noise components in order to examine how higher attention has an impact on Bitcoin price's discovery. By using Google Trends data for representing investor attention they decompose Bitcoin prices by a state space modeling (SSM) approach and use the Bandi and Russell (2006) decomposition for robustness check. They document that higher attention about Bitcoin is tied with higher probability of irrational uninformed trading appearance. Notably, attention is not related to more informed investors. Thereby, higher levels of attention result into higher noise in the Bitcoin price discovery process. From another perspective, Guegan and Renault (2020) make use of approximately one million messages on StockTwits to look into the nexus between investor sentiment and Bitcoin returns. Multivariate regressions and Granger causality tests are employed. Results display that higher pricing efficiency emerges when higher-frequency data (up to 15 minutes) are taken into consideration for estimations. It is argued that for these high frequencies the sentiment by StckTwits messages positively and significantly influences Bitcoin returns. This is more obvious during bubble periods. It should be noted that for lowerfrequency data this connection disappears. Despite reliable results during the bubble the impact is not large enough so as for investor sentiment to increase investors' profitability.

The COVID-19 pandemic has also brought about an increasing bulk of academic research about the impacts of the recent pandemic on cryptocurrencies. This forms the third relevant strand of literature in the present study. Empirical investigation of these linkages includes the papers of Conlon and McGee (2020), Goodell and Goutte (2021), Mariana et al. (2021), and Omane-Adjepong and Alagidede (2021). Goodell and Goutte (2021) reveal that during the COVID-19 pandemic and especially after April 5, 2020, more deaths by the disease led to higher Bitcoin values. This nexus is expressed by co-movements of Bitcoin with fatalities caused by COVID-19. When it comes to the study of Conlon and McGee (2020), they document that Bitcoin prices follow the same direction with the S\&P500 market values. Thereby, it is argued that Bitcoin cannot serve as an efficient hedger against movements of this stock index. It should be noted that the inclusion of Bitcoin in a portfolio that contains the S\&P500 asset leads to an increase in the portfolio's downside risk.

On the contrary, Mariana et al. (2021) provide evidence that Bitcoin and Ethereum could serve as safe-haven assets against the S\&P500 index during the pandemic. Furthermore, Ethereum is revealed to exhibit stronger safe haven capabilities than Bitcoin. By extending research on developing countries, Omane-Adjepong and Alagidede (2021) support that Bitcoin has served well as a hedge against traditional assets in African financial markets during the epidemic. Nevertheless, it has presented significantly lower hedging capabilities during the pandemic. To be more precise, Bitcoin is found to have failed in absorbing the investment risk of the currency markets in Nigeria and South Africa.

The third strand of principal importance to which this paper contributes is about the nexus between the COVID-19 disease and stock markets in general. Albulescu (2021), Ciner (2021), Rahman et al. (2021), Salisu and Vo (2020) and Topcu and Gulal (2020) are among the most significant papers in 
this domain of the literature. To be more precise, Albulescu (2021) investigates how official announcements about the new numbers of patients and deaths by COVID-19 influence financial volatility in US markets. Findings reveal that COVID-19 cases and deaths in the US as well as in a global context lead to higher volatility in stock markets. Moreover, Ciner (2021) looks into whether traditional assets such as currencies, bonds, and commodities could serve as predictors of US stock markets during the COVID-19 pandemic. The evidence presented indicates that high-quality bonds but also risky corporate bonds are useful for predictions of stock returns.

In a somewhat different approach, Rahman et al. (2021) document that the Australian stock market exhibited a negative reaction to news about expansion of the pandemic and reacted positively only to governmental announcements about helping to preserve employment. Furthermore, the least profitable portfolios of investment assets have been receivers of the most harmful impacts by the COVID-19 disease. By their own viewpoint, Salisu and Vo (2020) examine whether trends in health news influence the predictability of stock returns when taking into consideration the 20 countries most affected by the disease. A strong link is detected and health news are found to be good predictors of stock market values. Asymmetries caused by macroeconomic factors are also found to be influential. By focusing interest on developing economies, Topcu and Gulal (2020) reveal that the COVID-19 pandemic has constituted a negative factor that affected emerging stock markets during March and April 2020. Nevertheless, these impacts have rendered less intense since mid-April. Moreover, it is supported that markets of advanced economies have suffered in a much lower extent by the COVID-19 pandemic than developing ones.

The fourth strand of relevant literature has centered interest on the impacts of COVID-19 on commodities such as oil and gold. Adekoya and Oliyide (2021) look into whether volatility spillovers exist between commodity and financial assets. Oil, gold, stocks and Bitcoin are under scrutiny. The infectious disease index is found to be influential concerning such linkages, especially at lower and middle-level quantiles. Moreover, Mensi et al. (2020) examine the impacts of the COVID-19 disease on the multifractality of gold and oil prices based on upward and downward trends. Strong evidence of asymmetric multifractality is revealed. There is found to be higher efficiency in the downside trend for Brent oil while on the upside trend for gold. When it comes to Salisu et al. (2021), they argue that gold could serve as an efficient safe haven against crude oil price risks during the COVID-19 disease. Similar evidence is provided as concerns the hedging effectiveness of silver, platinum and palladium against oil alone or in portfolios.

\section{Data and Methodology}

Estimations are conducted concerning the period since the outburst of the COVID-19 pandemic (and the first publication of official data about the disease) up to the present. Thereby, the period under scrutiny spans from 22 January 2020 until 15 September 2020. This covers the first wave of the disease that has led to a new bear tendency in the markets of well-established financial markets. It is very interesting from an economic viewpoint to look into the determinants of the increase in market values of cryptocurrencies that emerged in tandem with the overall pessimistic market sentiment during this period.

In order to shed light on the impacts of the COVID-19 disease on financial markets, the highly innovative Twitter-based Market Uncertainty (TMU) Index This was developed by Thomas Renault (University Paris 1 Panthéon-Sorbonne) in conjunction with Scott R. Baker (Northwestern), Nicholas Bloom (Stanford) and Steve Davis (University of Chicago).This index has been constructed by extracting all messages sent on Twitter which contain keywords connected with uncertainty, such as: 'uncertain', 'uncertainly', 'uncertainties', 'uncertainty'. English-language tweets are taken into consideration.

Moreover, daily data about the market values of the highly representative S\&P500 index have been extracted from Datastream. Furthermore, the Bitcoin, Bitcoin Cash, and Dogecoin major 
cryptocurrencies are investigated. Their daily values have been downloaded from the coinmarketcap.com database. In order to enrich our investigation, the daily market values of the major commodity of WTI crude oil also have been considered. These data have also been available by Datastream. Data have been transformed into logarithmic differences in order to express returns of variables. Figure 1 displays the main variables adopted in relation with the Twitter-based market uncertainty measure.
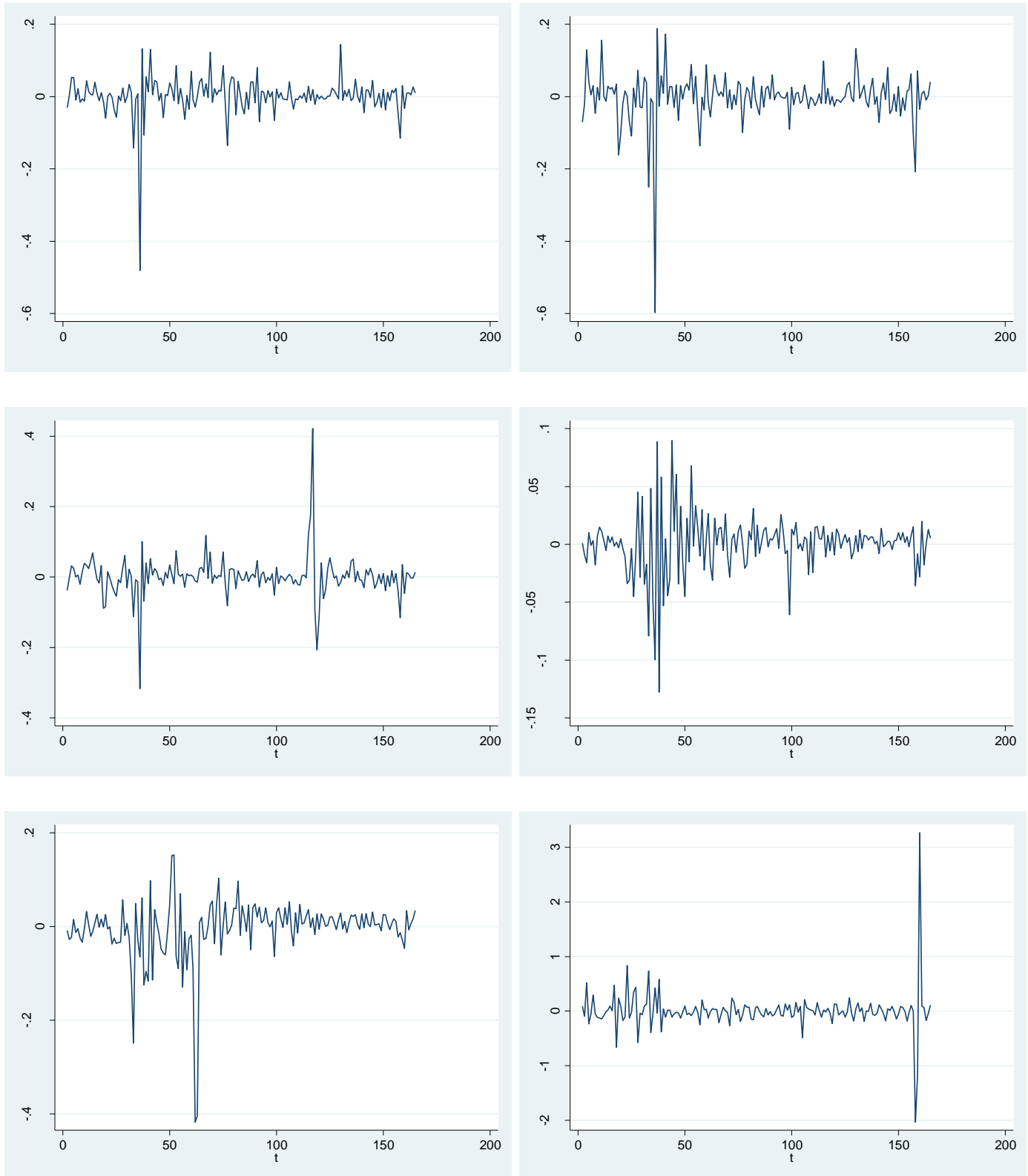

Figure 1: Returns of variables during the first 165 working days after the COVID-19 outburst (1st phase)

Table 1 displays the descriptive statistics of the variables under scrutiny. It can be easily seen that none of the cryptocurrencies examined has achieved large returns. Notably, Bitcoin Cash presents negative returns. This abides by the concept that the COVID-19 disease has caused bear tendencies in the major cryptocurrency markets. All the variables investigated present large levels of fluctuations and 
asymmetry due to conditions of high uncertainty prevailing. It should be noted that the Augmented Dickey-Fuller (ADF) as well as the Phillips-Perron tests provide evidence of stationarity in each of the variables examined.

Table 1: Descriptive statistics of returns of variables investigated.

\begin{tabular}{|c|c|c|c|c|c|c|c|c|}
\hline & Mean & $\begin{array}{l}\text { Std. } \\
\text { Dev. }\end{array}$ & Min & Max & Skewness & Kurtosis & ADF & $\mathbf{P P}$ \\
\hline BTC & 0.0013 & 0.0551 & $\begin{array}{c}- \\
0.4809 \\
\end{array}$ & 0.1444 & -4.043 & 38.053 & $\begin{array}{c}- \\
15.809 * * *\end{array}$ & $\begin{array}{c}- \\
15.537 * * *\end{array}$ \\
\hline $\mathbf{B C H}$ & $\begin{array}{c}- \\
0.0024 \\
\end{array}$ & 0.0723 & $\begin{array}{c}- \\
0.5977 \\
\end{array}$ & 0.1883 & -3.573 & 30.872 & $\begin{array}{c}- \\
14.752 * * * \\
\end{array}$ & $\begin{array}{c}- \\
14.674 * * *\end{array}$ \\
\hline DOGE & 0.0011 & 0.0586 & $\begin{array}{c}- \\
0.3169 \\
\end{array}$ & 0.4215 & 1.128 & 23.891 & $\begin{array}{c}- \\
11.374 * * * \\
\end{array}$ & $\begin{array}{c}- \\
11.328 * * *\end{array}$ \\
\hline S\&P500 & 0.0001 & 0.0262 & $\begin{array}{c}- \\
0.1277 \\
\end{array}$ & 0.0897 & -0.719 & 8.578 & $\begin{array}{c}- \\
18.705^{* * *}\end{array}$ & $\begin{array}{c}- \\
17.870 * * *\end{array}$ \\
\hline OIL & $\begin{array}{c}- \\
0.0046\end{array}$ & 0.0655 & $\begin{array}{c}- \\
0.4177 \\
\end{array}$ & 0.1529 & -3.184 & 20.535 & $-8.994 * * *$ & $-9.019 * * *$ \\
\hline TMU & 0.0048 & 0.3646 & $\begin{array}{c}- \\
2.0342\end{array}$ & 3.2632 & 3.217 & 46.616 & $\begin{array}{c}- \\
14.361 * * *\end{array}$ & $\begin{array}{c}- \\
16.313^{*} * *\end{array}$ \\
\hline
\end{tabular}

Table 2 provides the correlation matrix among market values of Bitcoin, Bitcoin Cash, and Dogecoin as well the S\&P500 representative index, crude oil and Twitter-based market uncertainty. Notably, it is easily discernible that all the assets examined display positive correlation coefficients in pairs with other assets and that the correlation coefficients between tech giant companies are medium to high. Crude oil is found to exhibit the lowest correlation with other assets and especially with cryptocurrencies. This informs the interested reader that diversification can take place in a portfolio consisting of digital currencies, the most representative and traditional stock index, and crude oil. Intriguingly, none of these assets seems to be appropriate for hedging against risk caused by the assets investigated. Moreover,, it should be emphasized that Twitter-based market uncertainty is negatively influential on the market returns of all assets examined. This reinforces the well-established concept that during uncertain times bear markets come to the surface in general.

Table 2: Correlation matrix of the variables investigated.

\begin{tabular}{|l|c|c|c|c|c|c|}
\hline & BTC & BCH & DOGE & S\&P500 & OIL & TMU \\
\hline BTC & 1.00 & & & & & \\
\hline BCH & 0.93 & 1.00 & & & & \\
\hline DOGE & 0.59 & 0.60 & 1.00 & & & \\
\hline S\&P500 & 0.51 & 0.48 & 0.35 & 1.00 & & \\
\hline OIL & 0.26 & 0.27 & 0.15 & 0.36 & 1.00 & \\
\hline TMU & -0.06 & -0.06 & -0.08 & -0.23 & -0.13 & 1.00 \\
\hline
\end{tabular}

We proceed to analyze the effects of stock indices, commodities, and the Twitter-based market uncertainty on high-capitalized cryptocurrency markets by employing two alternative sophisticated GARCH methodologies. Investigation also serves the purpose of identifying the pattern of impacts on returns and volatility that such important financial assets present. Therefore, empirical examination seeks to trace the existence or not of a non-linear character in the volatilities under scrutiny and whether this displays power patterns or not. To be more precise, the Non-linear GARCH and Non-linear Power 
GARCH methodologies are adopted in order to detect the exact nature of impacts that the representative stock index S\&P500, crude oil (that has indicates production growth and displayed large fluctuations in returns during the COVID-19 pandemic) and the Twitter-based investor sentiment index (which also exhibits large movements) have caused..

In order to examine whether stationarity exists the Dickey-Fuller and Phillips-Perron tests have been applied. Data have been transformed into logarithmic differences for the purposes of our estimations. It should be noted that two model selection criteria are used in order to make out the best fitting model for examining each of the financial assets considered. More specifically, the AIC (Akaike, 1974) and SBC/BIC (Schwarz, 1978) criteria are employed.

Emphasis should be put in that these advanced methodologies adopted are based on the ARCH (Engle, 1982) and GARCH (Bollerslev, 1986) models according to which the variance of the current error is a function of the volatility of the error conditions of previous time periods. The residual effect that has not been explained by alternative methodologies represents the error conditions in these specifications.

The ARCH methodology can be described by the following equation:

$$
h_{t}^{2}=\omega+\alpha u_{t-1}^{2}
$$

while the GARCH model is expressed as:

$$
h_{t}^{2}=\omega+\alpha u_{t-1}^{2}+\beta h_{t-1}^{2}
$$

Other GARCH-based schemes appropriate for detecting non-normality in volatility are employed:

Based on Higgins and Bera (1992) and Bollerslev et al. (1994), the non-linear GARCH and the non-linear Power GARCH models are adopted.

$$
\begin{gathered}
\text { Non - linear GARCH: } \sigma_{t}^{2}=\omega+\sum_{i=1}^{q} \alpha_{i}\left(\varepsilon_{t-i}-\kappa_{i}\right)^{2}+\sum_{j=1}^{p} \beta_{j} \sigma_{\tau-j}^{2} \\
\text { Non - linear Power GARCH: } \sigma_{t}^{\varphi}=\omega+\sum_{i=1}^{q} \alpha_{i}\left(\varepsilon_{t-i}-\kappa_{i}\right)^{\varphi}+\sum_{j=1}^{p} \beta_{j} \sigma_{\tau-j}^{2}
\end{gathered}
$$

Where $\omega$ is the constant of the variance equation. Moreover, $\alpha_{i}$ represents the ARCH term (the coefficient of squared lagged residuals) and indicates persistency of short-term shocks. Furthermore, $\beta_{j}$ stands for the GARCH term (the coefficient of lagged variance) and presents the existence of long-term shocks. Additionally, $\kappa_{i}$ illustrates non-linearity while $\varphi$ shows the power effect in volatility.

Abiding by the need to specify which methodology is more suitable, the Akaike Information Criterion (AIC) based on Akaike (1974) is applied:

$$
A I C=2 k-2 n L(\widehat{\Theta})
$$

Moreover, the Information Bayesian Criterion (SBC/BIC) based on Schwarz (1978) is adopted:

$$
B I C=k \ln n-2 \ln L(\widehat{\Theta})
$$

Where $k$ is the number of parameters estimated by the model, $n$ is the number of observations and $L(\hat{\Theta})$ represents the maximized value of the likelihood function of the model. The best fit model for the GARCH-type procedures conducted is given by the specification deriving the lowest AIC or BIC value. 


\section{Econometric outcomes}

Estimations have been conducted for investigating whether the S\&P500 index -which is highly representative of stock indices in a global context-, crude oil -that constitutes a significant indicator of economic activity- and the Twitter-based market sentiment -which forms an advanced measure of investor behaviour- affect the returns and volatility of Bitcoin. Non-linear GARCH and non-linear Power GARCH specifications have been adopted for the purposes of this investigation in order to detect the existence of non-linearities in the volatility of Bitcoin. The latter is the main driver in the markets of cryptocurrencies and the highest capitalized in the category of such financial assets. The same procedures have been followed in order to trace similar impacts on Bitcoin Cash and Dogecoin that are constantly among the highest-capitalized and most popular digital currencies. Investigation about non-linearities in major cryptocurrencies abides by previous research (Lahiani and Jlassi, 2021) but, to the best of our knowledge, the impact of the specific set of determinants has not been investigated earlier.

Estimations conducted by the Non-linear GARCH methodology are displayed in Table 3 and bring to the surface a number of fruitful findings. Evidence reveals that all three cryptocurrencies under scrutiny are receivers of significant positive effects from the S\&P500 index. More specifically, Bitcoin Cash is the most influenced (1.6009) while Bitcoin follows (1.0957) and Dogecoin is less affected (0.7865). All estimations are statistically significant at the $99 \%$ confidence interval. It should also be noted that crude oil prices prove to be influential by a weak level concerning these three cryptocurrencies. Arguably, Bitcoin is the receiver of the highest positive impact $(0.1388)$ whereas Bitcoin Cash is slightly less affected (0.0935). It is remarkable though that higher economic activity -as reflected by crude oil prices- exerts a negative impact on Dogecoin performance. Nevertheless, only the estimations about impacts on Bitcoin are trustworthy by a statistical perspective.

Interestingly, when it comes to results about the size and direction of Twitter-based market uncertainty, it can be observed that this is not much influential on the cryptocurrencies examined. Bitcoin Cash is revealed to be the most affected $(0.0243)$ by uncertainty as measured by tweets while Bitcoin (0.0136) and Dogecoin (0.0124) receive lower impacts. All estimations are statistically significant at least at the $90 \%$ confidence level. Emphasis should be given on that the parameters that indicate non-linearities in the volatility equation of each cryptocurrency are found to exhibit the highest level of statistical significance. This gives credence to the notion for existence of non-linear patterns in fluctuations of major digital currencies and paves the way for speculators to achieve high profits as future market prices are not easily predictable.

Table 3: Non-linear GARCH estimations

\begin{tabular}{|c|c|c|c|c|}
\hline & & Bitcoin & Bitcoin Cash & Dogecoin \\
\hline \multirow{4}{*}{$\begin{array}{l}\text { Mean } \\
\text { equation }\end{array}$} & S\&P500 & $1.0957 * * *$ & $1.6009 * * *$ & $0.7865 * * *$ \\
\hline & Oil & $0.1388 * * *$ & 0.0935 & -0.0076 \\
\hline & TMU & $0.0136^{*}$ & $0.0243 * *$ & $0.0124 * * *$ \\
\hline & Constant & 0.0023 & 0.0012 & 0.0007 \\
\hline \multirow{6}{*}{$\begin{array}{l}\text { Variance } \\
\text { equation }\end{array}$} & $\operatorname{Narch}(\alpha)$ & $0.6793 * * *$ & $0.7119 * * *$ & $0.7399 * * *$ \\
\hline & Narch_k (к) & $-0.0238 * * *$ & $-0.0302 * * *$ & $-0.0292 * * *$ \\
\hline & Garch $(\beta)$ & $0.2805^{* * *}$ & 0.0976 & $0.1028 *$ \\
\hline & Constant $(\omega)$ & $0.0003^{*}$ & $0.0011 * * *$ & $0.0002 *$ \\
\hline & AIC & -570.1943 & -466.0709 & -592.0147 \\
\hline & SBC/BIC & -545.3954 & -441.272 & -567.2158 \\
\hline
\end{tabular}

Note: $* * *, * * *$ display statistical significance at the $90 \%, 95 \%$, and $99 \%$ levels, respectively. Bold numbers indicate that the selection criteria exhibit lowest values achieved by this methodology. 
Furthermore, econometric outcomes based on the non-linear Power GARCH methodology indicate that Bitcoin is slightly more affected by the S\&P500 index (1.1468) in the same direction as nonlinear GARCH estimations reveal about volatility. Crude oil is found to be less influential (0.0287) as extracted by this specification while Twitter-based market uncertainty displays coefficient (0.0176) almost equal to previous estimations. All outcomes are statistically significant at least at the $90 \%$ confidence level. It should be emphasized that results are similar as concerns how these determinants influence Bitcoin Cash as provided by the estimates of impacts generated by the S\&P500 (1.0889), crude oil (0.0428), and Twitter-based investor sentiment concerning markets (0.0215). It should be stressed that the AIC and SBC/BIC model selection criteria give credence to the concept that non-linear Power GARCH is most preferable for estimations of effects on Bitcoin and Bitcoin Cash in relation to the nonlinear Power GARCH model.

Moreover, econometric evidence reveals that Dogecoin is a receiver of similar effects from the specific stock index, commodity, and investor sentiment index regardless of which of the specifications is employed for estimations. To be more precise, non-linear Power GARCH results exhibit coefficients for the S\&P500, crude oil, and TMU which are equal to $0.7703,-0.0073$, and 0.0132 , respectively. These are very close to previous findings. It is worth mentioning though that selection criteria give credence to the non-linear Power GARCH model being preferable for estimations. This supports the perspective that apart from the non-linear character in volatility, power patterns of volatility may emerge in this major cryptocurrency.

Table 4: Non-linear Power GARCH estimations

\begin{tabular}{|c|c|c|c|c|}
\hline & & Bitcoin & Bitcoin Cash & Dogecoin \\
\hline \multirow{4}{*}{$\begin{array}{l}\text { Mean } \\
\text { equation }\end{array}$} & S\&P500 & $1.1468 * * *$ & $1.0889 * * *$ & $0.7703 * * *$ \\
\hline & Oil & $0.0287 * *$ & $0.0428 * * *$ & -0.0073 \\
\hline & TMU & $0.0176 * * *$ & $0.0215 * * *$ & $0.0132 * * *$ \\
\hline & Constant & 0.0000 & 0.0004 & 0.0007 \\
\hline \multirow{4}{*}{$\begin{array}{l}\text { Variance } \\
\text { equation }\end{array}$} & NParch $(\alpha)$ & $0.1746^{* *}$ & $0.0847 *$ & $0.7798 * * *$ \\
\hline & NParch_k (к) & $-0.0126 * * *$ & $-0.0181 * * *$ & $-0.0293 * * *$ \\
\hline & 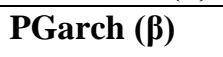 & $0.6816^{* * *}$ & $0.8346^{* * *}$ & 0.0598 \\
\hline & Constant $(\omega)$ & 0.2237 & 0.1206 & 0.0000 \\
\hline \multirow[t]{3}{*}{ Power } & Power $(\varphi)$ & -0.2261 & -0.3875 & $2.5676^{*}$ \\
\hline & AIC & -589.1838 & -507.1971 & -590.1664 \\
\hline & SBC/BIC & -561.285 & -479.2983 & -562.2676 \\
\hline
\end{tabular}

Note: $*, * *, * * *$ display statistical significance at the $90 \%, 95 \%$, and $99 \%$ levels, respectively. Bold numbers indicate that the selection criteria exhibit lowest values achieved by this methodology.

It can be observed that important economic implications are brought about by these econometric findings. Outcomes provide evidence that Bitcoin and Bitcoin Cash are influenced by the downwards tendencies in the market of the traditional and very representative S\&P500 stock index. Thereby, during abrupt decreases in market prices such as during the $1^{\text {st }}$ wave of the COVID-19 disease, major cryptocurrencies such as Bitcoin and Bitcoin Cash present movements in the same direction as and in a more or less equal extent with traditional stock markets. Notably, no significant levels of diversification or hedging can take place during such disease-led crises if the major stock indices are included in investor portfolios.

On the other hand, major cryptocurrencies are revealed to be influenced by traditional commodities - such as crude oil- by a very weak extent. One should bear in mind that oil is more than a 
simple commodity as is considered to represent economic activity. Estimations by both models display positive but weak impacts of oil on Bitcoin and Bitcoin Cash that are less powerful when non-linear Power GARCH estimations are performed. Somewhat surprisingly, the impacts on Dogecoin are negative and even weaker.

As concerns the way that Twitter-based market uncertainty influences major cryptocurrencies' performance, it is revealed that no strong effects are detected. This corroborates the view that Twitter sentiment is not able to influence the market behaviour of modern and sophisticated financial assets. Despite the anemic link presented by estimations of the nexus between tweets and cryptocurrencies, findings in the correlation matrix support that significant hedging effects could come to the surface between Twitter-based sentiment and traditional assets if examined. Therefore, research could focus on whether maturity in markets constitutes a significant parameter for influencing the level by which tweets could affect financial assets' performance.

\section{Conclusions}

Studying the impacts of major stock indices and commodities as well as Twitter-based market sentiment (TMU) on cryptocurrencies is of primary importance for enlightening investors regarding the factors that influence profit-making from modern financial assets. More specifically, the return and volatility features of largely-capitalized cryptocurrencies are examined in tandem with the impacts that traditional assets and sophisticated sentiment measures exert on them. This study contributes to academic literature about the linkages among major investment alternatives by the perspective of special volatility schemes. The second valuable contribution of this study is that this investigation is conducted through the lens of extremely high pressure in global economies during the first wave of the COVID-19 pandemic. The latter is considered to have been devastating for financial markets.

This paper sets under scrutiny the impacts that Bitcoin, Bitcoin Cash, and Dogecoin receive from the highly representative S\&P500 index, crude oil, and the market sentiment derived by tweets as expressed by the innovative Renault (2020) index. In order to detect non-linearities in fluctuations during the turbulent first wave of COVID-19, the non-linear GARCH and the non-linear Power GARCH specifications are adopted. The period examined spans from 22 January 2020 until 15 September 2020.

Econometric findings argue in favour of the S\&P500 index being substantially influential towards all three major cryptocurrencies in a positive manner. Crude oil -which represents economic activity- also exerts positive impacts on Bitcoin and Bitcoin Cash but in a much lower extent. Somewhat surprisingly, Dogecoin results do not comply with outcomes about Bitcoin and Bitcoin Cash as a negative though very weak coefficient is estimated. Emphasis should be given on that Twitter-based market uncertainty constitutes a positive determinant on cryptocurrencies but presents an anemic impact. This is in contrast with higher negative correlations between market uncertainty from tweets and traditional financial assets. Thereby it points towards the direction that less mature and more sophisticated assets are less affected by tweets.

It should be emphasized that Bitcoin and Bitcoin Cash are found to exhibit a non-linear GARCH pattern in their volatilities while the behaviour of Dogecoin is even more complex. The latter is better described by a non-linear Power GARCH specification. This points towards the notion that Dogecoin could prove more suitable for speculators in order to achieve higher profits. The reason for this is Dogecoin presenting a more complex volatility pattern that renders its returns less predictable for investors. Thereby, this would result into less forecasting accuracy and lower levels of efficiency in the Dogecoin market. This complexity leads to higher riskiness of this digital asset and larger levels of unpredictable volatility that could generate higher profits at the cost of higher uncertainty. Overall, results indicate that Dogecoin presents a weaker nexus with traditional assets and investor sentiment during the 
first wave of the COVID-19 pandemic. It could be argued that it constitutes a riskier asset than Bitcoin and Bitcoin Cash that could become more popular for speculators during a health crisis and a consequent financial crisis.

This study combines an overview of empirical studies on the nexus between Twitter-based market uncertainty and financial markets with an empirical investigation on the return and volatility impacts of this index and traditional assets on cryptocurrencies. This contributes to better understanding the determinants of effects on the most modern and popular financial assets for investors. Thereby, this paper sheds light on the size and direction of impacts of conventional investments and Twitter-based uncertainty on modern investments during crises such as the COVID-19 pandemic. This allows investors to enrich their arsenal of knowledge about decision-making in finance by incorporating the impact of investor sentiment on financial markets in their strategies. This is of primordial importance in the modern and highly-evolutionary financial environment that presents large masses of investors being susceptible to behavioural factors and mimicking behaviors. The large fluctuations in returns and volatility of both traditional and modern financial assets nowadays render essential the use of behavioural measures and the detection of non-linearities in volatilities in order to acquire a clearer view concerning investment decisions. This paper wishes to contribute even in the slightest degree towards this direction.

It can be concluded that information emanating from tweets has not become influential on the performance of cryptocurrencies while major stock indices exert positive and significant impacts and economic activity indicators such as oil are very weak determinants. This reveals that major cryptocurrencies are connected with financial markets in a much larger extent than with the real economy. This abides by their characterization as speculative assets. Potential avenues for further research in this field should include the examination of the nexus of a larger spectrum of cryptocurrencies and other sophisticated financial assets with alternative investments and sentiment indicators by employing more numerous and advanced methodologies.

\section{References}

Adekoya, O. B., \& Oliyide, J. A. (2021). How COVID-19 drives connectedness among commodity and financial markets: Evidence from TVP-VAR and causality-in-quantiles techniques. Resources Policy, 70, 101898.

Akaike, H. (1974). A new look at the statistical model identification. IEEE transactions on automatic control, 19(6), 716-723.

Albulescu, C. T. (2021). COVID-19 and the United States financial markets' volatility. Finance Research Letters, 38, 101699.

Ammous, S. (2018). Can cryptocurrencies fulfil the functions of money? The Quarterly Review of Economics and Finance, 70, 38-51.

Baig, A. S., Butt, H. A., Haroon, O., \& Rizvi, S. A. R. (2021). Deaths, panic, lockdowns and US equity markets: The case of COVID-19 pandemic. Finance research letters, 38, 101701.

Baig, A., Blau, B. M., \& Sabah, N. (2019). Price clustering and sentiment in bitcoin. Finance Research Letters, 29, 111-116.

Balcilar, M., Ozdemir, Z. A., Ozdemir, H., \& Wohar, M. E. (2020). Fed's unconventional monetary policy and risk spillover in the US financial markets. The Quarterly Review of Economics and Finance, 78, 42-52.

Beneki, C., Koulis, A., Kyriazis, N. A., \& Papadamou, S. (2019). Investigating volatility transmission and hedging properties between Bitcoin and Ethereum. Research in International Business and Finance, 48, 219-227.

Blau, B. M. (2018). Price dynamics and speculative trading in Bitcoin. Research in International Business and Finance, 43, 15-21. 
Böhme, R., Christin, N., Edelman, B., \& Moore, T. (2015). Bitcoin: Economics, technology, and governance. Journal of economic Perspectives, 29(2), 213-38.

Bollerslev, T. (1986). Generalized autoregressive conditional heteroskedasticity. Journal of econometrics, 31(3), 307-327.

Cheah, E. T., \& Fry, J. (2015). Speculative bubbles in Bitcoin markets? An empirical investigation into the fundamental value of Bitcoin. Economics letters, 130, 32-36.

Ciner, C. (2021). Stock Return Predictability in the time of COVID-19. Finance Research Letters, 38, 101705.

Conlon, T., \& McGee, R. (2020). Safe haven or risky hazard? Bitcoin during the COVID-19 bear market. Finance Research Letters, 35, 101607.

Corbet, S., Lucey, B., Urquhart, A., \& Yarovaya, L. (2019). Cryptocurrencies as a financial asset: A systematic analysis. International Review of Financial Analysis, 62, 182-199.

Diks, C., \& Panchenko, V. (2006). A new statistic and practical guidelines for nonparametric Granger causality testing. Journal of Economic Dynamics and Control, 30(9-10), 1647-1669.

Engle, R. F. (1982). Autoregressive conditional heteroscedasticity with estimates of the variance of United Kingdom inflation. Econometrica: Journal of the Econometric Society, 987-1007.

Eom, C., Kaizoji, T., Kang, S. H., \& Pichl, L. (2019). Bitcoin and investor sentiment: statistical characteristics and predictability. Physica A: Statistical Mechanics and its Applications, 514, 511521.

Fassas, A. P., Papadamou, S., \& Koulis, A. (2020). Price discovery in bitcoin futures. Research in International Business and Finance, 52, 101116.

Foley, S., Karlsen, J. R., \& Putniņš, T. J. (2019). Sex, drugs, and bitcoin: How much illegal activity is financed through cryptocurrencies?. The Review of Financial Studies, 32(5), 1798-1853.

Goodell, J. W., \& Goutte, S. (2021). Co-movement of COVID-19 and Bitcoin: Evidence from wavelet coherence analysis. Finance Research Letters, 38, 101625.

Granger, C. W. (1969). Investigating causal relations by econometric models and cross-spectral methods. Econometrica: Journal of the Econometric Society, 424-438.

Guégan, D., \& Renault, T. (2020). Does investor sentiment on social media provide robust information for Bitcoin returns predictability?. Finance Research Letters, 101494.

Higgins, M. L., \& Bera, A. K. (1992). A class of nonlinear ARCH models. International Economic Review, 137-158.

Huang, D., Jiang, F., Tu, J., \& Zhou, G. (2015). Investor sentiment aligned: A powerful predictor of stock returns. The Review of Financial Studies, 28(3), 791-837.

Ibikunle, G., McGroarty, F., \& Rzayev, K. (2020). More heat than light: Investor attention and bitcoin price discovery. International Review of Financial Analysis, 69, 101459.

Karalevicius, V., Degrande, N., \& De Weerdt, J. (2018). Using sentiment analysis to predict interday Bitcoin price movements. The Journal of Risk Finance, Vol. 19 No. 1, pp. 56-75.

Kyriazis, N., Papadamou, S., \& Corbet, S. (2020). A systematic review of the bubble dynamics of cryptocurrency prices. Research in International Business and Finance, 101254.

Lahiani, A., \& Jlassi, N. B. (2021). Nonlinear tail dependence in cryptocurrency-stock market returns: The role of Bitcoin futures. Research in International Business and Finance, 56, 101351.

Mariana, C. D., Ekaputra, I. A., \& Husodo, Z. A. (2021). Are Bitcoin and Ethereum safe-havens for stocks during the COVID-19 pandemic?. Finance research letters, 38, 101798.

Mensi, W., Sensoy, A., Vo, X. V., \& Kang, S. H. (2020). Impact of COVID-19 outbreak on asymmetric multifractality of gold and oil prices. Resources Policy, 69, 101829.

Omane-Adjepong, M., \& Alagidede, I. P. (2021). Exploration of safe havens for Africa's stock markets: A test case under COVID-19 crisis. Finance Research Letters, 38, 101877.

Papadamou, S., Kyriazis, N. A., \& Tzeremes, P. G. (2021). Non-linear causal linkages of EPU and gold with major cryptocurrencies during bull and bear markets. The North American Journal of Economics and Finance, 56, 101343. 
Philippas, D., Rjiba, H., Guesmi, K., \& Goutte, S. (2019). Media attention and Bitcoin prices. Finance Research Letters, 30, 37-43.

Rahman, M. L., Amin, A., \& Al Mamun, M. A. (2021). The COVID-19 outbreak and stock market reactions: Evidence from Australia. Finance Research Letters, 38, 101832.

Renault, T. (2020). Sentiment analysis and machine learning in finance: a comparison of methods and models on one million messages. Digital Finance, 2(1), 1-13.

Salisu, A. A., \& Vo, X. V. (2020). Predicting stock returns in the presence of COVID-19 pandemic: The role of health news. International Review of Financial Analysis, 71, 101546.

Salisu, A. A., Raheem, I. D., \& Vo, X. V. (2021). Assessing the safe haven property of the gold market during COVID-19 pandemic. International Review of Financial Analysis, 74, 101666.

Schwarz, G. (1978). Estimating the dimension of a model. Annals of statistics, 6(2), 461-464.

Shen, D., Urquhart, A., \& Wang, P. (2019). Does twitter predict Bitcoin?. Economics Letters, 174, 118122.

Topcu, M., \& Gulal, O. S. (2020). The impact of COVID-19 on emerging stock markets. Finance Research Letters, 36, 101691.

Yermack, D. (2015). Is Bitcoin a real currency? An economic appraisal. In Handbook of digital currency (pp. 31-43). Academic Press. 